\title{
BOUNDARY VALUE PROBLEMS OF HOLOMORPHIC VECTOR FUNCTIONS AND APPLICATIONS TO ANISOTROPIC ELASTICITY
}

\author{
BY \\ M. Z. WANG (Department of Mechanics, Peking University, Beijing, PR China) \\ A.ND \\ G. P. YAN (Department of Civil and Material Engineering, University of Illinois at Chicago)
}

\begin{abstract}
Using Stroh's formalism, plane problems of anisotropic elasticity are turned into the boundary value problems of holomorphic functions. A general method is presented for solving the boundary value problems. The displacement and the stress boundary value problems of an anisotropic body in an elliptical region are solved.
\end{abstract}

1. Introduction. The general solution to the class of problems in anisotropic materials in which the displacement and hence the stress are independent of the $x_{3}$-coordinate in a rectangular coordinate system was first obtained by Eshelby et al. [1]. This was extended by Stroh [2] who subsequently developed a powerful six-dimensional theory of dislocations and surface waves in anisotropic solids [3]. Stroh's theory has been further developed by Barnett and his co-workers in a series of papers [4-8]. An excellent review article on the theory of surface waves in anisotropic elastic materials was given by Chadwick and Smith [9]. Recent advances in the theory allow us to present certain aspects of the solutions in a real form [10-26].

Stroh's formalism transfers the stress or the displacement boundary value problems of a general anisotropic material to boundary value problems of holomorphic functions in a given region. Suo [18] solved several crack problems of holomorphic vector functions in terms of Hilbert problems. The purpose of this paper is to give another general method for solving the boundary value problems of the holomorphic vector functions, which are met in two-dimensional anisotropic elasticity. Then we apply the general method to some special problems. In Sec. 2 Stroh's formalism is outlined. Of several different notations found in the literature, we follow the notation employed in Ting [11]. In Sec. 3 the general method is given for solving the boundary value problems of holomorphic vector functions. In Sec. 4 a conformal mapping from an ellipse to an annulus is introduced. In Sec. 5, using the general methods of Secs. 2 and 3 the stress and the displacement boundary value problems of an ellipse of an anisotropic material are solved.

Received May 2, 1994.

1991 Mathematics Subject Classification. Primary 73B40.

(C)1997 Brown University 
Finally, in the Appendix the existence of inverses of some matrices is proved. It is important for the completeness of our paper. Inverses of some similar matrices were also met in [27], but their existence was not proved.

2. The Stroh formalism. In a fixed rectangular coordinate system $x_{i}(i=1,2,3)$, let $u_{i}, \sigma_{i j}$ be the displacement and stress, respectively. Assuming that $u_{i}(i=1,2,3)$ depend on $x_{1}$ and $x_{2}$, by the Stroh formalism, the general solution of two-dimensional anisotropic elasticity can be written in matrix notation (see Ting [11]) as

$$
\begin{aligned}
\mathbf{u} & =\mathbf{A} \mathbf{F}(z)+\overline{\mathbf{A}} \overline{\mathbf{F}(z)} \\
\boldsymbol{\varphi} & =\mathbf{B} \mathbf{F}(z)+\overline{\mathbf{B}} \overline{\mathbf{F}(z)}
\end{aligned}
$$

and

$$
\begin{gathered}
\mathbf{F}(z)=\left(F_{1}\left(z_{1}\right), F_{2}\left(z_{2}\right), F_{3}\left(z_{3}\right)\right)^{\mathrm{T}}, \\
z_{\alpha}=x_{1}+p_{\alpha} x_{2} \quad(\alpha=1,2,3),
\end{gathered}
$$

where $\mathbf{u}=\left(u_{1}, u_{2}, u_{3}\right)^{\mathrm{T}}$ and the superscript $\mathrm{T}$ stands for the transpose. In (2.1)-(2.4), $\mathbf{A}$ and $\mathbf{B}$ are $3 \times 3$ matrices, and $p_{\alpha}(\alpha=1,2,3)$ are complex constants, which are dependent on elastic constants. It is proved that $p_{\alpha}$ in (2.4) cannot be real if the strain energy is positive. In $(2.2), \varphi=\left(\varphi_{1}, \varphi_{2}, \varphi_{3}\right)^{\mathrm{T}}$ is a stress function vector, i.e.,

$$
\sigma_{i 1}=-\varphi_{i, 2}, \quad \sigma_{i 2}=\varphi_{i, 1} \quad(i=1,2,3)
$$

where a comma stands for differentiation. More generally, if $\tau$ is the surface traction at a point on a curved boundary,

$$
\tau=-\frac{\partial \varphi}{\partial s}
$$

where $s$ is the arc length along the curved boundary in the direction such that the region is located on the left-hand side [2] when one faces the direction of increasing $s$.

Thus, for the problem of two-dimensional anisotropic elasticity, what we need to do is to find a holomorphic vector function $\mathbf{F}(z)$ that satisfies a given boundary condition. In the next section, we will give a general method to find the holomorphic vector function $\mathbf{F}(z)$.

3. Boundary value problems of holomorphic vector functions. Let $\Gamma$ be a simple contour, $\Omega^{+}$and $\Omega^{-}$the interior and the exterior of $\Gamma$. Let the positive direction on $\Gamma$ be such that $\Omega^{+}$remains on the left. The contour $\Gamma$ will not be included in $\Omega^{+}$or $\Omega^{-}$. Further, let

$$
g(t)=g_{1}(t)+i g_{2}(t)
$$

be a continuous function given on $\Gamma$.

Consider the question as to whether $g(t)$ can be the boundary value of some function $F(\zeta)$, holomorphic in $\Omega^{+}$, where reference here is, of course, to boundary values as $\zeta \rightarrow t$ from $\Omega^{+}$. It is easily seen that, in general, it is impossible if the continuous function $g(t)$ is otherwise arbitrary. But, there is the following lemma [28]. 


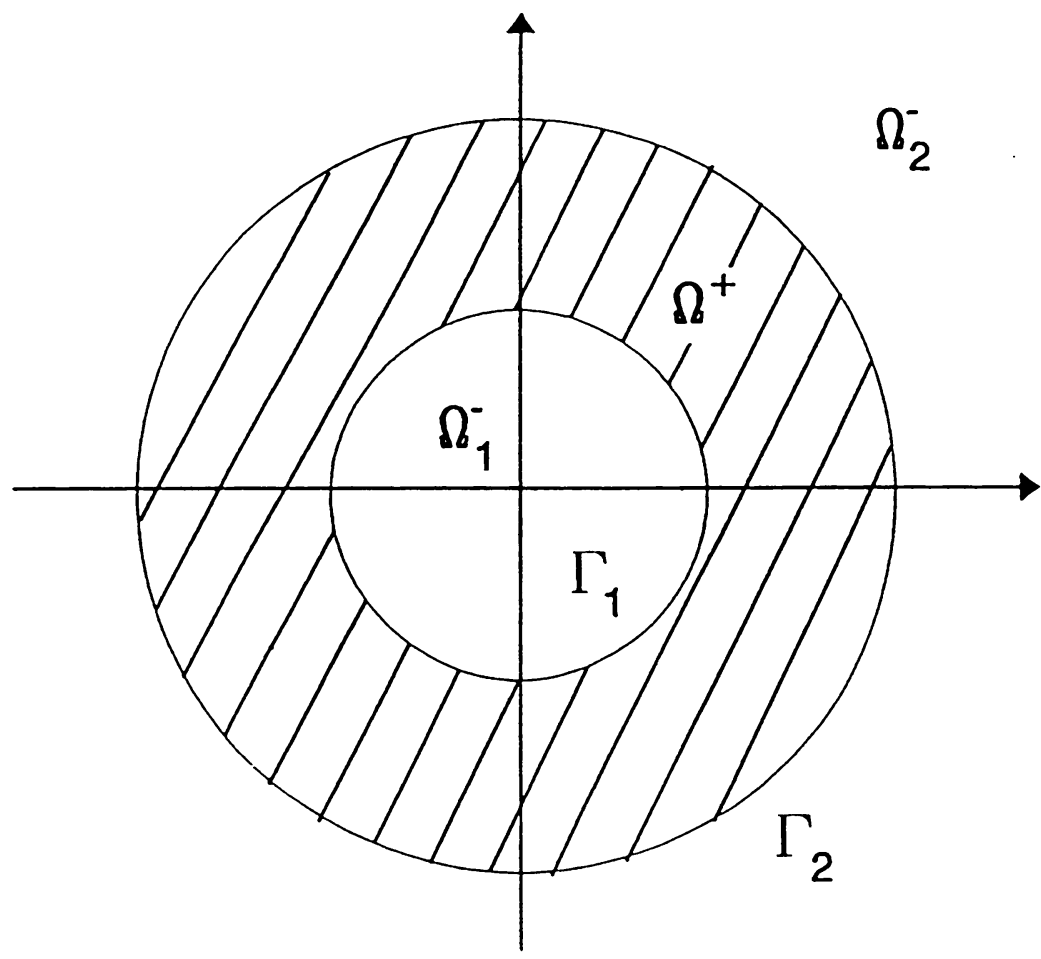

FIG. 1

LEMma. A necessary and sufficient condition for a continuous function $g(t)$, given on $\Gamma$, to be the boundary value of some function $F(\zeta)$, holomorphic in $\Omega^{+}$, is

$$
\frac{1}{2 \pi i} \oint_{\Gamma} \frac{g(t)}{t-\zeta} d t=0 \quad\left(\text { for all } \zeta \text { in } \Omega^{-}\right)
$$

and

$$
F(\zeta)=\frac{1}{2 \pi i} \oint_{\Gamma} \frac{g(t)}{t-\zeta} d t \quad\left(\zeta \text { in } \Omega^{+}\right) .
$$

In two-dimensional anisotropic elasticity we will study the following type of boundary value problems of a holomorphic vector function:

$$
\mathbf{B F}(t)+\overline{\mathbf{B}} \overline{\mathbf{F}(t)}=2 g_{1}(t) \mathbf{c} \quad(t \text { on } \Gamma),
$$

where

$$
\mathbf{F}(\zeta)=\left(F_{1}\left(\zeta_{1}\right), F_{2}\left(\zeta_{2}\right), F_{3}\left(\zeta_{3}\right)\right)^{\mathrm{T}},
$$

and $F_{\alpha}(\alpha=1,2,3)$ are holomorphic in some region $\Omega^{+}, \mathbf{B}$ is a constant matrix, $\mathbf{c}$ a constant vector, and $g_{1}(t)$ a known real function. Using the preceding lemma yields THEOREM. The holomorphic vector solution $\mathbf{F}(\zeta)$ in $\Omega^{+}$of Eq. (3.3) is

$$
\mathbf{F}(\zeta)=\left\langle\frac{1}{2 \pi i} \oint_{\Gamma} \frac{g_{1}(t)+i g_{2}(t)}{t-\zeta} d t\right\rangle \mathbf{B}^{-1} \mathbf{c} \quad\left(\zeta \text { in } \Omega^{+}\right)
$$


where \langle\rangle is a diagonal matrix, the elements of which are the values of $\zeta=\zeta_{1}, \zeta_{2}, \zeta_{3}$ (also see the second equation of (4.7)), and the real function $g_{2}(t)$ to be determined satisfies the following condition:

$$
\frac{1}{2 \pi i} \oint_{\Gamma} \frac{g_{1}(t)+i g_{2}(t)}{t-\zeta} d t=0 \quad\left(\text { for all } \zeta \text { in } \Omega^{-}\right)
$$

Proof. Let $\zeta \rightarrow t \in \Gamma$ from $\Omega^{+}$in (3.4). Since the condition (3.5) holds, the lemma may be used. Thus we can obtain

$$
\mathbf{F}(t)=\left(g_{1}(t)+i g_{2}(t)\right) \mathbf{B}^{-1} \mathbf{c}
$$

From (3.6) it is easy to know that $\mathbf{F}(\zeta)$ in (3.4) satisfies the boundary condition (3.3).

So far it has been assumed that the region $\Omega^{+}$is simply-connected. However, the preceding lemma and theorem can be extended to the case when the region $\Omega^{+}$is multiplyconnected. For simplicity assume that the region $\Omega^{+}$is doubly-connected, and bounded by two simple closed contours $\Gamma_{1}$ and $\Gamma_{2}$. The region inside the contour $\Gamma_{1}$ and the region outside the contour $\Gamma_{2}$ are denoted $\Omega_{1}^{-}$and $\Omega_{2}^{-}$(Fig. 1), respectively. Thus the lemma and the theorem still hold if we write $\Gamma_{1}+\Gamma_{2}$ for $\Gamma$ and $\Omega_{1}^{-}+\Omega_{2}^{-}$for $\Omega^{-}$.

4. Conformal mapping from an ellipse to an annulus. Let the boundary $L$ of an anisotropic ellipse $G$ be given by

$$
z=x_{1}+i x_{2}=a \cos \psi+i b \sin \psi
$$

where $a$ and $b$ are the major and minor semi-axes, respectively, and $\psi$ is a real parameter.

In the $z_{\alpha}$-plane, the corresponding ellipse is $G_{\alpha}$, and its boundary is

$$
L^{\alpha}: \quad z_{\alpha}=x_{1}+p_{\alpha} x_{2}=a \cos \psi+p_{\alpha} b \sin \psi
$$

Consider the mapping

$$
z_{\alpha}=\frac{1}{2}\left\{\left(a-i p_{\alpha} b\right) \zeta_{\alpha}+\left(a+i p_{\alpha} b\right) \zeta_{\alpha}^{-1}\right\}, \quad \alpha \text { not summed }
$$

or

$$
\zeta_{\alpha}=\frac{z_{\alpha}+\sqrt{z_{\alpha}^{2}-a^{2}-p_{\alpha}^{2} b^{2}}}{a-i p_{\alpha} b} .
$$

Equation (4.1) transforms the ellipse $G_{\alpha}$ in the $z_{\alpha}$-plane to an annulus $\Omega^{+}$in the $\zeta_{\alpha}$-plane,

$$
\Omega^{+}: \quad \rho_{\alpha} \leq|\zeta| \leq 1
$$

where

$$
\begin{gathered}
\rho_{\alpha}=\sqrt{\left|\lambda_{\alpha}^{+} / \lambda_{\alpha}^{-}\right|} \\
\lambda_{\alpha}^{+}=a+i b p_{\alpha} \quad \lambda_{\alpha}^{-}=a-i b p_{\alpha} .
\end{gathered}
$$


It has been shown [24] that $\rho_{\alpha}<1$. We see that the two points $\sqrt{\lambda_{\alpha}^{+} / \lambda_{\alpha}^{-}} e^{i \psi}$ and $\sqrt{\lambda_{\alpha}^{+} / \lambda_{\alpha}^{-}} e^{-i \psi}$ in the $\zeta$-plane correspond to the same point $\sqrt{\lambda_{\alpha}^{+} / \lambda_{\alpha}^{-}} \cos \psi$ in the $z_{\alpha^{-}}$ plane. After conformal mapping (4.1), the holomorphic function $F_{\alpha}\left(\zeta_{\alpha}\right)$ should satisfy the connected condition,

$$
F_{\alpha}\left(\sqrt{\lambda_{\alpha}^{+} / \lambda_{\alpha}^{-}} e^{i \psi}\right)=F_{\alpha}\left(\sqrt{\lambda_{\alpha}^{+} / \lambda_{\alpha}^{-}} e^{i \psi}\right) .
$$

The holomorphic vector function $\mathbf{F}(\zeta)$ in the annulus $\Omega^{+}$may be expanded in a Laurent series

$$
\mathbf{F}(\zeta)=\sum_{n=-\infty}^{n=+\infty}\left\langle\zeta^{n}\right\rangle \boldsymbol{\Lambda}_{-}^{n} \mathbf{q}_{n}
$$

where

$$
\begin{gathered}
\mathbf{\Lambda}_{-}=\left(\lambda_{1}^{-}, \lambda_{2}^{-}, \lambda_{3}^{-}\right)=a \mathbf{I}-i b \mathbf{P}, \\
\left\langle\zeta^{n}\right\rangle=\operatorname{diag}\left(\zeta_{1}^{n}, \zeta_{2}^{n}, \zeta_{3}^{n}\right), \\
\mathbf{P}=\operatorname{diag}\left(p_{1}, p_{2}, p_{3}\right) .
\end{gathered}
$$

Substitution of the series (4.6) into the connected condition (4.5) yields

$$
\mathbf{\Lambda}_{-}^{-n} \mathbf{q}_{-n}=\mathbf{\Lambda}_{+}^{n} \mathbf{q}_{n}
$$

where

$$
\boldsymbol{\Lambda}_{+}=\left(\lambda_{1}^{+}, \lambda_{2}^{+}, \lambda_{3}^{+}\right)=a \mathbf{I}+i b \mathbf{P}
$$

5. The ellipse subject to prescribed tractions. Consider the ellipse that is subject to an arbitrarily prescribed traction $\tau(\psi)$ on the boundary $L$. Let

$$
\rho(\psi) \tau(\psi)=\mathbf{s}_{0}+2 i \sum_{n=1}^{\infty} n\left(\mathbf{s}_{n} \sigma^{n}-\overline{\mathbf{s}}_{n} \sigma^{-n}\right)
$$

where

$$
\rho(\psi)=\sqrt{a^{2} \sin ^{2} \psi+b^{2} \cos ^{2} \psi}, \quad \sigma=e^{i \psi},
$$

and $\mathbf{s}_{n}$ are known complex constant vectors. Assume that the loading $\tau(\psi)$ is in equilibrium over the boundary, i.e.,

$$
\begin{gathered}
\int_{0}^{\psi} \rho(\psi) \tau(\psi) d \psi=0, \\
\int_{0}^{\psi} \rho(\psi) \tau(\psi) \times \mathbf{r}(\psi) \cdot \mathbf{k} d \psi=0,
\end{gathered}
$$

where $\mathbf{k}$ is the unit vector in the $x_{3}$-direction and $\mathbf{r}(\psi)$ the position vector

$$
\mathbf{r}(\psi)=(a \cos \psi, b \sin \psi, 0)^{\mathrm{T}} .
$$

Substituting (5.1) into (5.2) and (5.3), one yields

$$
\mathbf{s}_{0}=0, \quad b \operatorname{Re} s_{1}^{(1)}=a \operatorname{Im} s_{1}^{(2)},
$$


where $\mathbf{s}_{1}=\left(s_{1}^{(1)}, s_{2}^{(2)}, s_{3}^{(3)}\right)^{\mathrm{T}}$ and Re stands for the real part.

Integrating both sides of (2.6) along the boundary $L$ and using the formula $d s=$ $\rho(\psi) d \psi$, we obtain

$$
\varphi(\sigma)=-\int_{0}^{\psi} \rho(\psi) \tau(\psi) d \psi
$$

Substituting $(2.2),(5.1)$, and $(5.4)_{1}$ into the left side and right side of $(5.5)$, respectively, we obtain

$$
\mathbf{B F}(\sigma)+\overline{\mathbf{B}} \overline{\mathbf{F}(\sigma)}=-2 \sum_{n=1}^{\infty}\left(\mathbf{s}_{n} \sigma^{n}+\overline{\mathbf{s}}_{n} \sigma^{-n}\right) .
$$

In the derivation of (5.6) the condition $\mathbf{s}_{0}=0$ is used; otherwise, there would be a nonperiodic term in (5.6). Using the theorem in the preceding section, from (5.6) we know

$$
\mathbf{F}(\sigma)=-\mathbf{B}^{-1} \sum_{n=1}^{\infty}\left(\mathbf{s}_{n} \sigma^{n}+\overline{\mathbf{s}}_{n} \sigma^{-n}\right)+i \mathbf{B}^{-1} \mathbf{g}(\sigma),
$$

where the real function $\mathbf{g}(\sigma)$ is to be determined. Let

$$
i \mathbf{g}(\sigma)=\sum_{n=1}^{\infty}\left(\mathbf{k}_{n} \sigma^{n}-\overline{\mathbf{k}}_{n} \sigma^{-n}\right)
$$

where the complex constant $\mathbf{k}_{n}$ is to be determined. Substitution of (5.7) and (5.8) into (3.5) for the doubly-connected region $\Omega^{+}$in Fig. 1 yields

$$
\begin{aligned}
& \left\langle\frac{1}{2 \pi i} \oint_{\Gamma_{1}} \frac{t^{n}}{t-\zeta} d t\right\rangle \mathbf{\Lambda}_{-}^{n} \mathbf{q}_{n}+\left\langle\frac{1}{2 \pi i} \oint_{\Gamma_{2}} \frac{t^{n}}{t-\zeta} d t\right\rangle \mathbf{B}^{-1}\left(-\mathbf{s}_{n}+\mathbf{k}_{n}\right) \\
& \quad+\left\langle\frac{1}{2 \pi i} \oint_{\Gamma_{1}} \frac{t^{-n}}{t-\zeta} d t\right\rangle \mathbf{\Lambda}_{+}^{n} \mathbf{q}_{n}+\left\langle\frac{1}{2 \pi i} \oint_{\Gamma_{2}} \frac{t^{-n}}{t-\zeta} d t\right\rangle \mathbf{B}^{-1}\left(-\overline{\mathbf{s}}_{n}-\overline{\mathbf{k}}_{n}\right)=0
\end{aligned}
$$

(for all $\zeta$ in $\Omega_{1}^{-}$and $\Omega_{2}^{-}$).

When $\zeta$ is in $\Omega_{1}^{-}$, from (5.9) we obtain

$$
\mathbf{\Lambda}_{-}^{n} \mathbf{q}_{n}+\mathbf{B}^{-1}\left(-\mathbf{s}_{n}+\mathbf{k}_{n}\right)=0 .
$$

When $\zeta$ is in $\Omega_{2}^{-}$, from (5.9) we obtain

$$
\mathbf{\Lambda}_{+}^{n} \mathbf{q}_{n}+\mathbf{B}^{-1}\left(-\overline{\mathbf{s}}_{n}-\overline{\mathbf{k}}_{n}\right)=0 .
$$

Solving for $\mathbf{k}_{n}$ from (5.10),

$$
\mathbf{k}_{n}=-\mathbf{B} \boldsymbol{\Lambda}_{-}^{n} \mathbf{q}_{n}+\mathbf{s}_{n} .
$$

Substituting (5.12) into (5.11) yields

$$
\mathbf{B} \boldsymbol{\Lambda}_{+}^{n} \mathbf{q}_{n}+\overline{\mathbf{B}} \bar{\Lambda}_{-}^{n} \overline{\mathbf{q}}_{n}=2 \overline{\mathbf{s}}_{n} .
$$

Taking the conjugation of (5.13), we have

$$
\mathbf{B} \boldsymbol{\Lambda}_{-}^{n} \mathbf{q}_{n}+\overline{\mathbf{B}} \bar{\Lambda}_{+}^{n} \overline{\mathbf{q}}_{n}=2 \mathbf{s}_{n} .
$$


Equations (5.14) and (5.13) may be written

$$
\left[\begin{array}{ll}
\mathbf{B} \boldsymbol{\Lambda}_{-}^{n} & \overline{\mathbf{B}} \overline{\boldsymbol{\Lambda}}_{+}^{n} \\
\mathbf{B} \boldsymbol{\Lambda}_{+}^{n} & \overline{\mathbf{B}} \overline{\boldsymbol{\Lambda}}_{-}^{n}
\end{array}\right]\left[\begin{array}{l}
\mathbf{q}_{n} \\
\overline{\mathbf{q}_{n}}
\end{array}\right]=\left[\begin{array}{l}
2 \mathbf{s}_{n} \\
2 \overline{\mathbf{s}}_{n}
\end{array}\right]
$$

Eliminating $\overline{\mathbf{q}}_{n}$ in (5.15) gives

$$
\overline{\mathbf{K}}_{n} \mathbf{q}_{n}=-2 i\left(\overline{\boldsymbol{\Lambda}}_{-}^{n} \overline{\mathbf{B}}^{-1} \mathbf{s}_{n}-\overline{\mathbf{\Lambda}}_{+}^{n} \overline{\mathbf{B}}^{-1} \overline{\mathbf{s}}_{n}\right),
$$

where

$$
\mathbf{K}_{n}=\boldsymbol{\Lambda}_{-}^{n}\left(i \mathbf{B}^{-1} \overline{\mathbf{B}}\right) \overline{\mathbf{\Lambda}}_{-}^{n}-\boldsymbol{\Lambda}_{+}^{n}\left(i \mathbf{B}^{-1} \overline{\mathbf{B}}\right) \overline{\mathbf{\Lambda}}_{+}^{n} .
$$

In the Appendix we will prove that the coefficient matrices $\mathbf{K}_{n}$ are nonsingular for $n \geq 2$. Thus from (5.15) or (5.16) the constant vectors $\mathbf{q}_{n}(n \geq 2)$ can be solved.

For $n=1,(5.14)$ and (5.13) or (5.15) become

$$
\left\{\begin{array}{l}
\mathbf{B} \boldsymbol{\Lambda}_{-} \mathbf{q}_{1}+\overline{\mathbf{B}} \overline{\boldsymbol{\Lambda}}_{+} \overline{\mathbf{q}}_{1}=2 \mathbf{s}_{1} \\
\mathbf{B} \boldsymbol{\Lambda}_{+} \mathbf{q}_{1}+\overline{\mathbf{B}} \overline{\boldsymbol{\Lambda}}_{-} \overline{\mathbf{q}}_{1}=2 \overline{\mathbf{s}}_{1}
\end{array}\right.
$$

Noticing (4.7) and (4.9), we have from (5.18)

$$
\left\{\begin{array}{l}
a\left(\mathbf{B} \mathbf{q}_{1}+\overline{\mathbf{B}} \overline{\mathbf{q}}_{1}\right)=\mathbf{s}_{1}+\overline{\mathbf{s}}_{1}, \\
i b\left(\mathbf{B} \mathbf{P} \mathbf{q}_{1}+\overline{\mathbf{B}} \overline{\mathbf{P}} \overline{\mathbf{q}}_{1}\right)=-\left(\mathbf{s}_{1}-\overline{\mathbf{s}}_{1}\right),
\end{array}\right.
$$

or

$$
\left\{\begin{array}{l}
\operatorname{Re}\left(\mathbf{B} \mathbf{q}_{1}\right)=\frac{1}{a} \operatorname{Re} \mathbf{s}_{1}, \\
\operatorname{Re}\left(\mathbf{B} \mathbf{P} \mathbf{q}_{1}\right)=\frac{1}{b} \operatorname{Im} \mathbf{s}_{1},
\end{array}\right.
$$

For the stress function $\varphi$, the $n=1$ term denoted by $\varphi_{1}$ is

$$
\varphi_{1}=2 \operatorname{Re} \mathbf{B}\langle z\rangle \mathbf{q}_{1}
$$

which corresponds to a constant stress state. Using (5.19), the stress function (5.20) becomes

$$
\varphi_{1}=\frac{2 x_{1}}{a} \operatorname{Re} \mathbf{s}_{1}-\frac{2 x_{2}}{b} \operatorname{Im} \mathbf{s}_{1}
$$

which gives the constant stress

$$
\left\{\begin{array}{l}
\sigma_{i 1}=-\varphi_{1,2}^{(i)}=\frac{2}{b} \operatorname{Im} s_{1}^{(i)} \\
\sigma_{i 2}=\varphi_{1,1}^{(i)}=\frac{2}{a} \operatorname{Re} s_{1}^{(i)}
\end{array}\right.
$$

where $\varphi_{1}=\left(\varphi_{1}^{(1)}, \varphi_{1}^{(2)}, \varphi_{1}^{(3)}\right)^{\mathrm{T}}$. Since $\sigma_{12}=\sigma_{21},(5.22)$ leads to $(5.4)_{2}$. (Note: The conditions that the resultant moments in the $x_{1}$ - and $x_{2}$-directions vanish are not necessary for the existence of a solution to the problem. Similar situations were found for anisotropic elastic wedges subject to a concentrated couple [13] and for the anisotropic elastic semi-infinite strip [25].)

The solution of the displacement boundary value problem of an anisotropic ellipse is similar to the stress boundary value problem if we replace the matrix $\mathbf{B}$ by $\mathbf{A}$ in the section. 
6. Discussion and concluding remarks. Stroh's formalism transfers boundary value problems of a general anisotropic material to boundary value problems of holomorphic functions in a given region. We present a general method for solving the boundary value problem of holomorphic functions if the conformal mapping from regions in the planes $z_{\alpha}$ to the unit circle in the plane $\zeta_{\alpha}$ is known. Unfortunately, as Gao pointed out in [29], up to now there are only two known conformal mappings, i.e., for an ellipse and for a half-plane, associated with Stroh's formalism.

Acknowledgments. The authors are greatly indebted to Prof. T. C. T. Ting, of University of Illinois at Chicago, for most valuable discussions and several illuminating comments. We would like to thank the reviewer for many useful suggestions.

\section{Appendix.}

Theorem. Matrices $\mathbf{K}_{n}(n \geq 2)$ are positive Hermitian, where

$$
\begin{gathered}
\mathbf{K}_{n}=\boldsymbol{\Lambda}_{-}^{n} \mathbf{F} \overline{\boldsymbol{\Lambda}}_{-}^{n}-\boldsymbol{\Lambda}_{+}^{n} \mathbf{F} \overline{\mathbf{\Lambda}}_{+}^{n}, \\
\boldsymbol{\Lambda}_{+}=a \mathbf{I}+i b \mathbf{P}, \\
\boldsymbol{\Lambda}_{-}=a \mathbf{I}-i b \mathbf{P}, \\
\mathbf{P}=\operatorname{diag}\left(p_{1}, p_{2}, p_{3}\right), \\
\mathbf{F}=i \mathbf{B}^{-1} \overline{\mathbf{B}} .
\end{gathered}
$$

First introduce some lemmas.

Lemma 1. F is positive Hermitian, i.e.,

$$
-i \mathbf{B}^{\mathrm{T}} \overline{\mathbf{B}}^{-\mathrm{T}}=i \mathbf{B}^{-1} \overline{\mathbf{B}}
$$

The proof of Lemma 1 can be found [22].

Lemma 2. $\mathbf{C}^{*} \mathbf{G C}$ is positive or semi-positive Hermitian if $\mathbf{G}$ is positive or semi-positive Hermitian and $\mathbf{C}$ is a nonsingular matrix, where the superscript ${ }^{*}$ stands for the transpose and the complex conjugate.

Lemma 2 is obvious. Now we come to prove the following lemma.

Lemma $3 . \mathbf{K}_{1}$ is semi-positive Hermitian, and the null vector of $\mathbf{K}_{1}$ is $\overline{\mathbf{B}}^{\mathrm{T}} \mathbf{J}$.

Proof. Substituting (A.1) 2,3 into (A.1) 1 for $n=1$ yields

$$
\begin{aligned}
\mathbf{K}_{1} & =\boldsymbol{\Lambda}_{-} \mathbf{F} \overline{\boldsymbol{\Lambda}}_{-}-\boldsymbol{\Lambda}_{+} \mathbf{F} \overline{\boldsymbol{\Lambda}}_{+} \\
& =(a \mathbf{I}-i b \mathbf{P}) \mathbf{F}(a \mathbf{I}+i b \overline{\mathbf{P}})-(a \mathbf{I}+i b \mathbf{P}) \mathbf{F}(a \mathbf{I}-i b \overline{\mathbf{P}}) \\
& =2 i a b(\mathbf{F} \overline{\mathbf{P}}-\mathbf{P} \mathbf{F}) .
\end{aligned}
$$

Using (A.1) $)_{5}$ and Lemma 1, (A.2) becomes

$$
\mathbf{K}_{1}=-2 a b \mathbf{B}^{-1}\left(\overline{\mathbf{B}} \overline{\mathbf{P}} \overline{\mathbf{B}}^{\mathrm{T}}+\mathbf{B} \mathbf{P} \mathbf{B}^{\mathrm{T}}\right) \overline{\mathbf{B}}^{-\mathrm{T}}
$$


According to [19],

$$
\overline{\mathbf{B}} \overline{\mathbf{P}} \overline{\mathbf{B}}^{\mathrm{T}}+\mathbf{B P B}^{\mathrm{T}}=\mathbf{N}_{3} \text {. }
$$

It is shown in [19] that $-\mathbf{N}_{3}$ is positive semi-definite, and its rank is two and $-\mathbf{N}_{3}$ has the following form:

$$
-\mathbf{N}_{3}=\left[\begin{array}{lll}
* & 0 & * \\
0 & 0 & 0 \\
* & 0 & *
\end{array}\right]
$$

where the $*$ denotes a possibly nonzero element. From (A.5) we know the null vector of $\mathbf{N}_{3}$ is

$$
\mathbf{J}=(0,1,0)^{\mathrm{T}}
$$

Thus from (A.3), (A.5), and Lemma 2, Lemma 3 is proved.

LEMMA 4.

$$
\mathbf{K}_{n+1}=a^{2} \mathbf{K}_{n}+b^{2} \mathbf{P} \mathbf{K}_{n} \overline{\mathbf{P}}+\frac{1}{2}\left(\boldsymbol{\Lambda}_{-} \mathbf{K}_{1} \overline{\boldsymbol{\Lambda}}_{-}^{n}+\boldsymbol{\Lambda}_{+}^{n} \mathbf{K}_{1} \overline{\boldsymbol{\Lambda}}_{+}^{n}\right) .
$$

Proof. From $(\text { A.1 })_{1}$ we have

$$
\mathbf{K}_{n+1}=(a \mathbf{I}-i b \mathbf{P}) \boldsymbol{\Lambda}_{-}^{n} \mathbf{F} \overline{\boldsymbol{\Lambda}}_{-}^{n}(a \mathbf{I}+i b \overline{\mathbf{P}})-(a \mathbf{I}+i b \mathbf{P}) \boldsymbol{\Lambda}_{+}^{n} \mathbf{F} \overline{\mathbf{\Lambda}}_{+}^{n}(a \mathbf{I}-i b \overline{\mathbf{P}}),
$$

or

$$
\begin{aligned}
\mathbf{K}_{n+1}=a^{2} & \left\{\boldsymbol{\Lambda}_{-}^{n} \mathbf{F} \bar{\Lambda}_{-}^{n}-\boldsymbol{\Lambda}_{+}^{n} \mathbf{F} \bar{\Lambda}_{+}^{n}\right\}+b^{2} \mathbf{P}\left\{\boldsymbol{\Lambda}_{-}^{n} \mathbf{F} \bar{\Lambda}_{-}^{n}-\boldsymbol{\Lambda}_{+}^{n} \mathbf{F} \bar{\Lambda}_{+}^{n}\right\} \overline{\mathbf{P}} \\
& -i a b\left\{\mathbf{P} \boldsymbol{\Lambda}_{-}^{n} \mathbf{F} \bar{\Lambda}_{-}^{n}-\boldsymbol{\Lambda}_{-}^{n} \mathbf{F} \bar{\Lambda}_{-}^{n} \overline{\mathbf{P}}+\mathbf{P} \boldsymbol{\Lambda}_{+}^{n} \mathbf{F} \bar{\Lambda}_{+}^{n}-\boldsymbol{\Lambda}_{+}^{n} \mathbf{F} \bar{\Lambda}_{+}^{n} \overline{\mathbf{P}}\right\} .
\end{aligned}
$$

Since $\boldsymbol{\Lambda}_{+}, \boldsymbol{\Lambda}_{-}$, and $\mathbf{P}$ commute, (A.8) leads to (A.7).

Lemma 5. $\mathbf{K}_{2}$ is positive Hermitian.

Proof. For $n=1$, (A.7) gives

$$
\mathbf{K}_{2}=a^{2} \mathbf{K}_{1}+b^{2} \mathbf{P} \mathbf{K}_{1} \overline{\mathbf{P}}+\frac{1}{2}\left(\boldsymbol{\Lambda}_{-} \mathbf{K}_{1} \overline{\boldsymbol{\Lambda}}_{-}+\boldsymbol{\Lambda}_{+} \mathbf{K}_{1} \overline{\boldsymbol{\Lambda}}_{+}\right)
$$

In order to prove the lemma it will be sufficient from Lemma 3 and (A.9) that $\mathbf{K}_{1}$ and $\mathbf{P K}_{1} \overline{\mathbf{P}}$ have no common null vector.

Suppose $\mathbf{K}_{1}$ and $\mathbf{P} \mathbf{K}_{1} \overline{\mathbf{P}}$ have a common null vector. From Lemma 3 , we know that the null vectors of $\mathbf{K}_{1}$ and $\mathbf{P} \mathbf{K}_{1} \overline{\mathbf{P}}$ are, respectively,

$$
\overline{\mathbf{B}}^{\mathrm{T}} \mathbf{J} \text { and } \overline{\mathbf{P}}^{-1} \overline{\mathbf{B}}^{\mathrm{T}} \mathbf{J}
$$

where $\mathbf{J}$ is defined in (A.6). This means

$$
\overline{\mathbf{B}}^{\mathrm{T}} \mathbf{J}=\bar{\lambda} \overline{\mathbf{P}}^{-1} \overline{\mathbf{B}}^{\mathrm{T}} \mathbf{J}
$$

or

$$
\mathbf{B}^{\mathrm{T}} \mathbf{J}=\lambda \mathbf{P}^{-1} \mathbf{B}^{\mathrm{T}} \mathbf{J}
$$

or

$$
\left[\begin{array}{l}
B_{21} \\
B_{22} \\
B_{23}
\end{array}\right]=\lambda\left[\begin{array}{c}
\frac{1}{p_{1}} B_{21} \\
\frac{1}{p_{2}} B_{22} \\
\frac{1}{p_{3}} B_{23}
\end{array}\right] .
$$


It is assumed in this paper that $p_{1}, p_{2}, p_{3}$ are distinct. Thus (A.10) holds, when only one of $B_{21}, B_{22}, B_{23}$ is nonzero. Since [2],

$$
B_{1 \alpha}=-p_{\alpha} B_{2 \alpha} \quad(\alpha=1,2,3)
$$

if $B_{21}$ is the only nonzero element,

$$
\mathbf{B}=\left[\begin{array}{ccc}
-p_{1} B_{21} & 0 & 0 \\
B_{21} & 0 & 0 \\
B_{31} & B_{32} & B_{33}
\end{array}\right],
$$

which is singular. This leads to a contradiction since $\mathbf{B}$ is nonsingular. The same contradiction is obtained if $B_{22}$ or $B_{23}$ is the only nonzero element.

Proof of the theorem. Since $\mathbf{K}_{2}$ is positive Hermitian, we assume that $\mathbf{K}_{n}(n \geq 2)$ is positive Hermitian. From the recurrence formula (A.7) we obtain that $\mathbf{K}_{n+1}$ will also be positive Hermitian. By the mathematical induction method, $\mathbf{K}_{n}(n \geq 2)$ are all positive Hermitian.

\section{REFERENCES}

[1] J. D. Eshelby, W. T. Read, and W. Shockley, Anisotropic elasticity with applications to dislocation theory, Acta Metallurgica 1, 251-259 (1953)

[2] A. N. Stroh, Dislocations and cracks in anisotropic elasticity, Philos. Mag. 3, 625-646 (1958)

[3] A. N. Stroh, Steady state problems in anisotropic elasticity, J. Math. Phys. 41, 77-103 (1962)

[4] D. M. Barnett and J. Lothe, Synthesis of the sextic and the integral formalism for dislocations, Green's function and surface waves in anisotropic elastic solids, Phys. Norv. 7, 13-19 (1973)

[5] D. M. Barnett and J. Lothe, An image force theorem for dislocations in anisotropic bicrystals, J. Phys. Fluids 4, 1618-1635 (1974)

[6] D. M. Barnett and J. Lothe, Line force loadings on anisotropic half-spaces and wedges, Phys. Norv. 8, 13-22 (1985)

[7] D. M. Barnett and J. Lothe, Free surface (Rayleigh) waves in anisotropic elastic half-spaces: The surface impedance method, Proc. Royal Soc. London Ser. A 402, 135-152 (1985)

[8] R. J. Asaro, J. P. Hirth, D. M. Barnett, and J. Lothe, A further synthesis of sextic and integral theories for dislocations and line forces in anisotropic media, Phys. Status Solidi B 60, 261-271 (1973)

[9] P. Chadwick and G. D. Smith, Foundations of the theory of surface waves in anisotropic elastic materials, Adv. Appl. Mech. 17, 303-376 (1977)

[10] H. O. K. Kirchner and J. Lothe, Displacements and tractions along interfaces, Philos. Mag. A 56, 583-594 (1987)

[11] T. C. T. Ting, Explicit solution and invariance of the singularities at an interface crack in anisotropic composites, Internat. J. Solids Structures 22, 965-983 (1986)

[12] T. C. T. Ting, Line forces and dislocations in anisotropic elastic composite wedges and spaces, Phys. Status Solidi B 146, 81-90 (1988)

[13] T. C. T. Ting, The anisotropic elastic wedge under a concentrated couple, Quart. J. Mech. Appl. Math. 41, 562-578 (1988)

[14] R. Chadwick, Wave propagation in transversely isotropic elastic media. I. Homogeneous plane waves. II. Surface waves. III. The special case $a_{s}=0$ and the inextensible limit, Proc. Roy. Soc. London Ser. A 422, 23-121 (1989)

[15] Chyanbin Hwu and T. C. T. Ting, Two-dimensional problems of the anisotropic elastic solids with an elliptic inclusion, Quart. J. Mech. Appl. Math. 42, 553-572 (1989)

[16] Qianqian Li and T. C. T. Ting, Line inclusions in anisotropic elastic solids, J. Appl. Mech. 56, $556-563$ (1989)

[17] Jianmin Qu and Qianqian $\mathrm{Li}$, Interfacial dislocation and its application to interface crack in anisotropic materials, J. Elasticity 26, 169-195 (1991) 
[18] Zhigang Suo, Singularities, interfaces and cracks in dissimilar anisotropic media, Proc. Roy. Soc. London Ser. A 427, 331-358 (1991)

[19] T. C. T. Ting, Some identities and the structure of $\mathbf{N}_{i}$ in the Stroh formalism of anisotropic elasticity, Quart. Appl. Math. 46, 109-120 (1988)

[20] T. C. T. Ting, Effects of change of reference coordinates on the stress analyses of anisotropic elastic materials, Internat. J. Solids Structures 18, 139-152 (1982)

[21] T. C. T. Ting and Chyanbin Hwu, Sextic formalism in anisotropic elasticity for almost nonsemisimple matrix N, Internat. J. Solids Structures 24, 65-76 (1988)

[22] T. C. T. Ting, On the orthogonal, Hermitian and positive definite properties of the matrices $i B^{-1} \bar{B}$ and $-i A^{-1} \bar{A}$ in anisotropic elasticity, J. Elasticity 30, 277-284 (1993)

[23] T. C. T. Ting, Barnett-Lothe tensors and their associated tensors for monoclinic materials with the symmetry plane at $x_{3}=0$, J. Elasticity 27, 143-165 (1992)

[24] T. C. T. Ting and G. P. Yan, The anisotropic elastic solid with an elliptic hole or rigid inclusion, Internat. J. Solids Structures 27, 1879-1894 (1991)

[25] T. C. T. Ting and M. Z. Wang, Generalized Stroh formalism for anisotropic elasticity for general boundary conditions, Acta Mech. Sinica 8, 193-207 (1992)

[26] M. Z. Wang, T. C. T. Ting, and G. P. Yan, The anisotropic elastic semi-infinite strip, Quart. Appl. Math. 51, 283-297 (1993)

[27] S. G. Lekhnitskii, Anisotropic Plate, Gordon and Breach Science Publishers, 1968

[28] N. I. Muskhelishvili, Some Basic Problems of the Mathematical Theory of Elasticity, Noordhoff, 1963

[29] H. J. Gao, Stress of holes in anisotropic elastic solids: conformal mapping and boundary perturbation, Quart. J. Mech. Appl. Math. 45, 149-168 (1992) 